PROCEEDINGS OF THE

AMERICAN MATHEMATICAL SOCIETY

Volume 132, Number 6, Pages 1627-1637

S 0002-9939(04)07315-0

Article electronically published on January 22, 2004

\title{
DECAY OF POSITIVE WAVES FOR $n \times n$ HYPERBOLIC SYSTEMS OF BALANCE LAWS
}

\author{
PAOLA GOATIN AND LAURENT GOSSE
}

(Communicated by Suncica Canic)

\begin{abstract}
We prove Olĕnik-type decay estimates for entropy solutions of $n \times n$ strictly hyperbolic systems of balance laws built out of a wave-front tracking procedure inside which the source term is treated as a nonconservative product localized on a discrete lattice.
\end{abstract}

\section{INTRODUCTION}

A classical result proved by Oleĭnik [18] for strictly convex scalar conservation laws in one space dimension shows that the density of positive waves decays in time like $\mathcal{O}(1 / t)$, see also [14]. More precisely, if we consider the scalar equation

$$
u_{t}+f(u)_{x}=0, \quad u(t=0, \cdot)=u_{o} \in \mathbf{L}^{\infty}(\mathbb{R}),
$$

with $f^{\prime \prime}(u) \geq \kappa>0$, we have that every entropy-admissible solution satisfies

$$
u(t, y)-u(t, x) \leq \frac{y-x}{\kappa t} \quad \text { for all } t>0, x<y,
$$

and therefore has locally bounded variation (see also [8], Theorem 11.2.2). Conversely, if $u=u(t, x)$ is a weak solution satisfying (1.2), then $u$ is entropy admissible.

The same estimate as in (1.2) has been recovered for the Riemann coordinates of a particular $2 \times 2$ system [4] and for $n \times n$ genuinely nonlinear systems belonging to the Temple class 7 . However, one cannot expect such a result to remain valid for general $n \times n$ systems, even assuming that all characteristic fields are genuinely nonlinear. Indeed, interactions among existing shocks may generate rarefactions as time increases. Decay estimates must therefore take into account the generation of new positive waves due to interactions. Results in this direction were proved by Liu [17 in the case of approximate solutions constructed by Glimm's scheme [9], and by Bressan and Colombo [5] for exact solutions obtained as limits of front tracking appoximations for $n \times n$ homogeneous systems, [19]. This in turn yields uniqueness of solutions satisfying the Oleı̆nik entropy condition ([6], [7, [10]). From the point of view of practical applications, such one-sided estimates are very useful, for instance, in the context of multiphase geometric optics computations [12], or local error estimates [20, and asymptotic behaviour of entropy solutions.

Received by the editors December 21, 2001.

2000 Mathematics Subject Classification. Primary 35L65; Secondary 35L45.

Key words and phrases. Conservation laws, source terms, nonconservative products.

The authors were partially supported respectively by the EC-Marie Curie Individual Fellowship \#HPMF-CT-2000-00930 and EEC grants \#ERBFMRXCT970157 \& \#HPRN-CT-2002-00282.

(C)2004 American Mathematical Society 
In this paper we are interested in extending Oleunik-type estimates on positive waves to quasilinear systems of balance laws. More precisely, we shall deal with the Cauchy problem for the following $n \times n$ system of equations:

$$
u_{t}+f(u)_{x}=g(x, u), \quad x \in \mathbb{R}, t>0,
$$

endowed with a (suitably small) initial data $u_{o} \in \mathbf{L}^{1} \cap \mathbf{B V}\left(\mathbb{R} ; \mathbb{R}^{n}\right)$. Here $u(t, x) \in \mathbb{R}^{n}$ is the unknown function, and $f: \Omega \rightarrow \mathbb{R}^{n}$ is a smooth $\mathcal{C}^{2}$ vector field defined on an open neighborhood $\Omega$ of the origin in $\mathbb{R}^{n}$. We will assume that the system (1.3) is strictly hyperbolic, with each characteristic field either genuinely nonlinear or linearly degenerate in the sense of Lax [15. Moreover, we assume the following Carathéodory-type conditions for the source term $g$ :

(P1) $g: \mathbb{R} \times \Omega \rightarrow \mathbb{R}^{n}$ is measurable w.r.t. $x$, for any $u \in \Omega$, and is $\mathcal{C}^{2}$ w.r.t. $u$, for any $x \in \mathbb{R}$;

(P2) $\|g(x, \cdot)\|_{\mathcal{C}^{2}}$ is bounded over $\Omega$, uniformly in $x \in \mathbb{R}$;

(P3) there exists a function $\omega \in \mathbf{L}^{1}(\mathbb{R}) \cap \mathbf{L}^{\infty}(\mathbb{R})$ such that $|g(x, u)| \leq \omega(x)$ and $\left\|\nabla_{u} g(x, u)\right\| \leq \omega(x)$ for all $(x, u) \in \mathbb{R} \times \Omega$.

In addition, we require that a non-resonance condition holds; that is, the characteristic speeds are bounded away from zero: for some $p \in\{1, \ldots, n\}$ and some $c>0$ one has

$$
\begin{cases}\lambda_{i}(u) \leq-c & \text { if } i \leq p, \\ \lambda_{i}(u) \geq c & \text { if } i>p\end{cases}
$$

for all $u \in \Omega$, where $\lambda_{i}(u)$ denote the eigenvalues of the Jacobian matrix $D f(u)$.

Under these assumptions, it was proved in [1] that there exists a family of entropy weak solutions to (1.3) continuously depending on the initial data. More precisely, if the $\mathbf{L}^{1}$-norm of $\omega$ is small enough, there exist a closed domain $\mathcal{D} \subset \mathbf{L}^{1}\left(\mathbb{R} ; \mathbb{R}^{n}\right)$ of functions with sufficiently small total variation, a constant $L$ and a unique semigroup $P:[0,+\infty) \times \mathcal{D} \rightarrow \mathcal{D}$ with the properties:

(i) For all $u, v \in \mathcal{D}$ and $t, s \geq 0$ one has $\left\|P_{s} u-P_{t} v\right\|_{\mathbf{L}^{1}} \leq L\left(|t-s|+\|u-v\|_{\mathbf{L}^{1}}\right)$.

(ii) For all $u_{o} \in \mathcal{D}$ the function $u(t, \cdot)=P_{t} u_{o}$ is a weak entropy solution of the Cauchy problem (1.3), $u(t=0, \cdot)=u_{o}$.

Under the above assumptions, we aim to show that, for genuinely nonlinear characteristic fields, an Oleŭnik type estimate on the decay of positive waves holds, which takes into account not only new waves generated by interactions but even the contribution of the source term. A careful statement of these results requires some notation ([3], [5], 6]).

As usual, let $A(u)=D f(u)$ be the Jacobian matrix of $f$, and call $\lambda_{i}(u), l_{i}(u)$, $r_{i}(u)$ respectively the eigenvalues and the left and right eigenvctors of $A(u)$. Let $u$ : $\mathbb{R} \rightarrow \Omega$ have bounded variation in $x$ and satisfy (1.3) with $g \equiv 0$. The distributional derivative $\mu \doteq D_{x} u$ is a vector measure. For $i=1, \ldots, n$ we can now define $\mu^{i}$ as

$$
\int \phi d \mu^{i}=\int \phi \tilde{l}_{i} \cdot D_{x} u, \quad \phi \in \mathcal{C}_{c}^{0},
$$

where $\tilde{l}_{i}(x)=l_{i}(u(x))$ at points where $u$ is continuous, while $\tilde{l}_{i}\left(x_{\alpha}\right)$ is some vector that satisfies

$$
\begin{gathered}
\left|\tilde{l}_{i}\left(x_{\alpha}\right)-l_{i}\left(u\left(x_{\alpha}\right)\right)\right|=\mathcal{O}(1) \cdot\left|u\left(x_{\alpha}+\right)-u\left(x_{\alpha}-\right)\right|, \\
\tilde{l}_{i}\left(x_{\alpha}\right) \cdot\left(u\left(x_{\alpha}+\right)-u\left(x_{\alpha}-\right)\right)=\sigma_{\alpha}^{i},
\end{gathered}
$$


where $\sigma_{\alpha}^{i}$ denotes the strength of the $i$-th wave generated by the resolution of the corresponding discontinuity in $x_{\alpha}$.

We denote by $\mu^{i+}, \mu^{i-}$ the positive and negative parts of $\mu$; then we have

$$
\mu^{i}=\mu^{i+}-\mu^{i-}, \quad\left|\mu^{i}\right|=\mu^{i+}+\mu^{i-} .
$$

The total strength of waves in $u$ is defined as

$$
\mathbf{V}(u) \doteq \sum_{i=1}^{n} \mathbf{V}_{i}(u), \quad \mathbf{V}_{i}(u) \doteq\left|\mu^{i}\right|(\mathbb{R}),
$$

while the interaction potential is defined in terms of product measures on $\mathbb{R}^{2}$ :

$$
\mathbf{Q}(u) \doteq \sum_{i<j}\left(\left|\mu^{j}\right| \times\left|\mu^{i}\right|\right)(\{(x, y): x<y\})+\sum_{i \in \mathcal{G} \mathcal{N}}\left(\mu^{i-} \times\left|\mu^{i}\right|\right)(\{(x, y): x \neq y\}),
$$

where $\mathcal{G N}$ denotes the set of genuinely nonlinear families.

Now we are ready to state our main result in the case $g \not \equiv 0$ :

Theorem 1.1 (Decay of positive waves). Let the system (1.3) be strictly hyperbolic and let the $i$-th characteristic field be genuinely nonlinear. Then there exists a constant $C$, depending solely on $f$, such that for every $0 \leq s<t$ and every solution $u$ with small total variation obtained as a limit of wave-front tracking approximations, the measure $\mu_{t}^{i+}$ of $i$-waves in $u(t, \cdot)$ satisfies

$$
\mu_{t}^{i+}(J) \leq C \cdot\left(\frac{\operatorname{meas}(J)}{t-s}+\mathbf{Q}(s)-\mathbf{Q}(t)+\mathbf{V}\left(u_{o}\right) \cdot\|\omega\|_{\mathbf{L}^{1}}\right)
$$

for every Borel set $J \subset \mathbb{R}$.

Of course, we tacitly assume (P1)-(P3) and (1.4) throughout this paper.

\section{WAVE-FRONT TRACKING WITH ZERO-WAVES}

In this section we briefly recall the construction of wave-front tracking approximations as stated in 1. We start with the definition of the $h$-Riemann solver. For small $h>0$ we introduce the map

$$
\Phi_{h}\left(x_{o}, u\right) \doteq f^{-1}\left[f(u)+\int_{0}^{h} g\left(x_{o}+s, u\right) d s\right]
$$

(note that $f$ is invertible due to (1.4)), which approximates the flow of the stationary equation associated to (1.3). Consider now the Riemann problem with initial states

$$
u(0, x)= \begin{cases}u_{l} & \text { if } x<x_{o}, \\ u_{r} & \text { if } x>x_{o}\end{cases}
$$

To locally render the source term's effects, a stationary discontinuity is introduced along the line $x=x_{o}$, that is, a wave whose speed is equal to zero; it will be referred to as a zero-wave. An $h$-Riemann solver for (1.3) - 2.1) has been defined in [1] as a self-similar function $u(t, x)=R_{h}\left(\left(x-x_{o}\right) / t ; u_{l}, u_{r}\right)$ as follows:

(a) there exist two states $u^{-}, u^{+}$that satisfy $u^{+}=\Phi_{h}\left(x_{o}, u^{-}\right)$;

(b) $u(t, x)$ coincides, on the set $\left\{t \geq 0, x<x_{o}\right\}$, with the solution to the homogeneous Riemann problem with initial values $u_{l}, u^{-}$and, on the set $\left\{t \geq 0, x>x_{o}\right\}$, with the solution to the homogeneous Riemann problem with initial values $u^{+}, u_{r}$; 
(c) the Riemann problem between $u_{l}$ and $u^{-}$is solved only by waves with negative speed (i.e., of the families $1, \ldots, p$ );

(d) the Riemann problem between $u^{+}$and $u_{r}$ is solved only by waves with positive speed (i.e., of the families $p+1, \ldots, n$ ).

This clearly shares a lot of common features with the nonconservative Riemann problems studied in [16]. Now let $\epsilon, h>0$ be given: an $(\epsilon, h)$-approximate solution of (1.3) is constructed as follows. First of all, the source term is localized by means of a Dirac comb along zero-waves located on the lattice $x=j h, j \in\left(-\frac{1}{h \epsilon}, \frac{1}{h \epsilon}\right) \cap \mathbb{Z}$ :

$$
u_{t}+f(u)_{x}=h \sum_{j} g(x, u) \cdot \delta(x-j h),
$$

where $\delta$ stands for the Dirac measure concentrated on $x=0$.

Given the initial data $u_{o}$, we deduce a piecewise constant approximation $u(0, \cdot)$ without increasing its $\mathbf{B V}$-norm, and $u(t, x)$ is constructed, for small $t$, by applying the $h$-Riemann solver at every point $x=j h$, and by solving the remaining discontinuities in $u(0, \cdot)$ using a classical homogeneous Riemann solver (rarefaction waves are discretized following [3]: for a fixed small parameter $\nu$, each rarefaction of size $\sigma$ is divided, at its starting time, into $m=\left[\frac{\sigma}{\nu}\right]+1$ wave fronts of size $\sigma / m \leq \nu$ ).

At every interaction point, a new Riemann problem arises. Notice that because of their null speed, zero-waves cannot interact with each other. In order to keep finite the total number of wave fronts, two distinct procedures are used for solving a Riemann problem: an accurate method, which possibly creates several new fronts, and a simplified method, which minimizes the number of new wave fronts. For a detailed description, as well as the proof of the stability of the algorithm, we refer the reader to [1, 3].

The approximate solution can have four types of jumps: shocks (or contact discontinuities), rarefaction fronts, non-physical waves and zero-waves: $\mathcal{J}=\mathcal{S} \cup$ $\mathcal{R} \cup \mathcal{N} \mathcal{P} \cup \mathcal{Z}$. A priori bounds on the functions $u^{\epsilon, h}$ are obtained by slightly modifying the Glimm's functionals [9] in order to keep track of the zero-waves,

$$
\begin{gathered}
V(t)=\sum_{\alpha \in \mathcal{J}}\left|\sigma_{\alpha}\right|=\sum_{\alpha \in \mathcal{S} \cup \mathcal{R} \cup \mathcal{N} \mathcal{P} \cup \mathcal{Z}}\left|\sigma_{\alpha}\right|, \\
Q(u(t))=\sum_{\alpha, \beta \in \tilde{\mathcal{A}}}\left|\sigma_{\alpha} \sigma_{\beta}\right| \leq V(t)^{2},
\end{gathered}
$$

measuring respectively the total wave strengths and the interaction potential in $u(t, \cdot)$. In particular, if $\alpha \in \mathcal{Z}$, then the strength of the wave located in $x_{\alpha}=j_{\alpha} h$ can be measured by means of

$$
\sigma_{\alpha}=\int_{0}^{h} \omega\left(j_{\alpha} h+s\right) d s .
$$

In (2.4) we have denoted by $\tilde{\mathcal{A}}$ an extended set of approaching waves. As usual, we call $k_{\alpha}$ the family of the front located at $x_{\alpha}$, with size $\sigma_{\alpha}$. More precisely, a pair of wave-fronts of families $k_{\alpha}, k_{\beta}$, located at $x_{\alpha}<x_{\beta}$, belongs to $\tilde{\mathcal{A}}$ in any of the following cases:

- if neither of the two is a zero-wave, either $k_{\alpha}>k_{\beta}$, or else $k_{\alpha}=k_{\beta}$ and at least one of them is a genuinely nonlinear shock, [9];

- if $\alpha$ is a zero-wave and $\beta$ is a physical one, $k_{\beta} \leq p$;

- if $\beta$ is a zero-wave and $\alpha$ is a physical one, $k_{\alpha}>p$. 
Notice that for some $C>1$,

$$
\frac{1}{C}\left[\|\omega\|_{\mathbf{L}^{1}(I)}+\operatorname{Tot} . \operatorname{Var} . u(t, \cdot)\right] \leq V(t) \leq C\left[\|\omega\|_{\mathbf{L}^{1}(I)}+\operatorname{Tot} . \operatorname{Var} . u(t, \cdot)\right],
$$

where the interval $I$ is defined by

$$
I \doteq \bigcup_{\alpha \in \mathcal{Z}}\left[j_{\alpha} h,\left(j_{\alpha}+1\right) h\right]=\bigcup_{j \in\left(-\frac{1}{\epsilon h}, \frac{1}{\epsilon h}\right) \cap \mathbb{Z}}[j h,(j+1) h] .
$$

Passing to the limit as $\epsilon \rightarrow 0, h>0$ fixed, one has:

(i) the total variation of $u^{\epsilon, h}(t, \cdot)$ remains uniformly bounded;

(ii) the maximum size of rarefaction fronts approaches zero;

(iii) the total strength of all non-physical waves approaches zero.

By (i), Helly's theorem guarantees the existence of a subsequence strongly convergent in $\mathbf{L}^{1}{ }_{\text {loc }}$. By (ii) and (iii), this limit provides a weak solution to (2.2) in agreement with nonconservative theories, [16]. At this stage, we can again extract a subsequence $u^{h_{i}}$ that converges to some function $u$ in $\mathbf{L}^{1}$ loc and solves (1.3) in the usual weak distributional sense.

In the sequel, we will need a semicontinuity property of Glimm's functionals. For $h>0$ fixed, let the total strength of waves $\mathbf{V}_{h}$ and the interaction potential $\mathbf{Q}_{h}$ be as in 1], Section 4.1; that is, they are defined as in (1.5)-(1.6), but including the zero-waves. We have

$$
\begin{gathered}
\mathbf{V}_{h}(u)=\mathbf{V}(u)+\|\omega\|_{\mathbf{L}^{1}}, \\
\mathbf{Q}(u) \leq \mathbf{Q}_{h} \leq \mathbf{Q}(u)+\|\omega\|_{\mathbf{L}^{1}} \cdot \mathbf{V}(u) .
\end{gathered}
$$

Proceeding as in [3], we recover the lower semicontinuity of the functionals $\mathbf{Q}_{h}$ and $\boldsymbol{\Upsilon}_{h}(u) \doteq \mathbf{V}_{h}(u)+C_{o} \mathbf{Q}_{h}(u), C_{o}>0$, on a domain $\mathcal{D}$ of the form

$$
\mathcal{D} \doteq\left\{u \in \mathbf{L}^{1} \cap \mathbf{B V}\left(\mathbb{R} ; \mathbb{R}^{n}\right), \Upsilon_{h}(u) \leq \gamma\right\}, \quad \gamma \text { small enough }
$$

(see [3], Theorem 10.1):

Theorem 2.1 (Lower semicontinuity of the Glimm functionals). There exists a choice of the constants $C_{o}, \gamma>0$ such that, if $\mathbf{\Upsilon}_{h}(u)=\mathbf{V}_{h}(u)+C_{o} \mathbf{Q}_{h}(u)<\gamma$, then for any sequence of functions $u_{\nu} \in \mathcal{D}, u_{\nu} \rightarrow u$ in $\mathbf{L}^{1}$ as $\nu \rightarrow \infty$, one has

$$
\begin{aligned}
& \mathbf{Q}_{h}(u) \leq \liminf _{\nu \rightarrow \infty} \mathbf{Q}_{h}\left(u_{\nu}\right), \\
& \boldsymbol{\Upsilon}_{h}(u) \leq \liminf _{\nu \rightarrow \infty} \boldsymbol{\Upsilon}_{h}\left(u_{\nu}\right) .
\end{aligned}
$$

Moreover, for every finite union of open intervals $J=I_{1} \cup \ldots \cup I_{S}$, one has

$$
\mu^{i \pm}(J)+C_{o} \mathbf{Q}_{h}(u) \leq \liminf _{\nu \rightarrow \infty}\left(\mu_{\nu}^{i \pm}(J)+C_{o} \mathbf{Q}_{h}\left(u_{\nu}\right)\right), \quad i=1, \ldots, n .
$$

\section{Proof of Theorem 1.1}

I. By Lipschitz continuous dependence of the trajectories it is not restrictive to assume $s=0, t=T$. We will consider a particular converging sequence $u^{\nu, h}$ of $\left(\epsilon_{\nu}, h\right)$-approximate solutions with the following properties:

(i) each rarefaction front $x_{\alpha}$ travels with the characteristic speed of the state on the right:

$$
\dot{x}_{\alpha}=\lambda_{k_{\alpha}}\left(u\left(x_{\alpha}+\right)\right)
$$


(ii) each shock $x_{\alpha}$ travels with a speed strictly contained between the right and the left characteristic speeds:

$$
\lambda_{k_{\alpha}}\left(u\left(x_{\alpha}+\right)\right)<\dot{x}_{\alpha}<\lambda_{k_{\alpha}}\left(u\left(x_{\alpha}-\right)\right) ;
$$

(iii) calling $N_{\nu}$ the number of jumps in $u_{o}^{\nu, h}=u^{\nu, h}(0, \cdot)$, as $\nu \rightarrow \infty$ one has

$$
\epsilon_{\nu} \rightarrow 0, \quad \epsilon_{\nu} N_{\nu} \rightarrow 0
$$

(iv) the interaction potential satisfies

$$
\mathbf{Q}_{h}\left(u^{\nu, h}(0, \cdot)\right) \rightarrow \mathbf{Q}_{h}\left(u_{o}\right) \quad \text { as } \nu \rightarrow \infty .
$$

Such a sequence can be constructed as explained in [3] (proof of Lemma 10.2, p. 205).

Let $u=u(t, x)$ be a piecewise constant $(\epsilon, h)$-approximate solution constructed via front-tracking approximation (we shall drop the $\epsilon, h$ superscripts since there is no ambiguity). As usual, by (generalized) $i$-characteristic we mean an absolutely continuous curve $x=x(t)$ such that, [8],

$$
\dot{x}(t) \in\left[\lambda_{i}(u(t, x+)), \lambda_{i}(u(t, x-))\right], \quad \text { a.e. } t \geq 0 .
$$

By $t \mapsto y^{i}(t ; \bar{x})$ we denote the minimal $i$-characteristic passing through $\bar{x}$ at time $T$. Because of (1.4) the presence of zero-waves does not affect the usual construction.

Now let $I \doteq[a, b[$ be any half-open interval, and define

$$
I(t) \doteq\left[y^{i}(t ; a), y^{i}(t ; b)[\doteq[a(t), b(t)[.\right.
$$

We seek an estimate of the number of positive $i$-waves in the approximate solution $u(T, \cdot)$ contained in $I$. We recall that $k_{\alpha}$ stands for the family of the front located at $x_{\alpha}$, with size $\sigma_{\alpha}$. For a genuinely nonlinear family, the size of the jump can be measured by

$$
\sigma_{\alpha} \doteq \lambda_{k_{\alpha}}\left(u\left(x_{\alpha}+\right)\right)-\lambda_{k_{\alpha}}\left(u\left(x_{\alpha}-\right)\right),
$$

while the size of a zero-wave is still given by (2.5). Define

$$
m(t) \doteq b(t)-a(t)
$$

By (3.3) and the Lipschitz continuity of the map $u \mapsto \lambda_{i}(u)$ we deduce that

$$
\begin{aligned}
\dot{m}(t) & =\lambda_{i}(u(t, b(t)))-\lambda_{i}(u(t, a(t))) \\
& =M(t)+\mathcal{O}(1)(\epsilon+K(t))
\end{aligned}
$$

for a.e. $t$. The Landau symbol stands for a quantity whose modulus is uniformly bounded. We see that

$$
M(t) \doteq \sum_{k_{\alpha}=i, x_{\alpha} \in I(t)} \sigma_{\alpha}=\mu_{t}^{i}(I(t))
$$

is the total number of (signed) $i$-waves in $u(t, \cdot)$ contained in $I(t)$, while

$$
K(t) \doteq \sum_{k_{\alpha} \neq i, x_{\alpha} \in I(t)}\left|\sigma_{\alpha}\right|=\sum_{k \neq i}\left|\mu_{t}^{k}\right|(I(t))+\int_{I(t)} \omega(x) d x
$$

stands for the total strength of waves of families $\neq i$ inside $I(t)$, zero-waves included. To estimate the contribution of the term $K(t)$ in (3.4) we introduce

$$
\Phi(t) \doteq \sum_{k_{\alpha} \neq i} \phi_{k_{\alpha}}\left(t, x_{\alpha}(t)\right) \cdot\left|\sigma_{\alpha}\right| \leq \mathbf{V}_{h}(u(t)),
$$


where

$$
\phi_{j}(t, x) \doteq \begin{cases}1 & \text { if } x<a(t), \\ \frac{b(t)-x}{m(t)} & \text { if } x \in[a(t), b(t)[ \\ 0 & \text { if } x \geq b(t),\end{cases}
$$

or

$$
\phi_{j}(t, x) \doteq \begin{cases}0 & \text { if } x<a(t), \\ \frac{x-a(t)}{m(t)} & \text { if } x \in[a(t), b(t)[, \\ 1 & \text { if } x \geq b(t),\end{cases}
$$

in the cases $j<i$ or $j>i$, respectively. Roughly speaking, $\Phi(t)$ represents the accumulated strength of the waves that do not approach the interval $I(t)$. By strict hyperbolicity, we can expect it to grow with time. Observe that $\Phi$ is piecewise Lipschitz continuous with a finite number of discontinuities occurring at interaction times, where it may decrease by at most

$$
\Phi(\tau+)-\Phi(\tau-)=\mathcal{O}(1)[Q(u(\tau-))-Q(u(\tau+))] .
$$

Following [3], we assume that, for some $c_{o}>0$,

$$
\left|\lambda_{i}(u)-\lambda_{i}(v)\right| \leq c_{o}, \quad\left|\lambda_{i}(u)-\lambda_{k_{\alpha}}(v)\right| \geq 2 c_{o},
$$

for every two states $u, v$ and every $k_{\alpha} \neq i$. Outside interaction times, $\Phi$ is nondecreasing; indeed, we have

$$
\begin{aligned}
\dot{\Phi}(t)= & \sum_{k_{\alpha} \neq i}\left|\sigma_{\alpha}\right| \cdot \frac{d}{d t} \phi_{k_{\alpha}}\left(t, x_{\alpha}(t)\right) \\
= & \sum_{k_{\alpha}<i, x_{\alpha} \in I(t)}\left|\sigma_{\alpha}\right| \cdot\left(\frac{\dot{b}-\dot{x}_{\alpha}}{m}-\frac{\left(b-x_{\alpha}\right) \dot{m}}{m^{2}}\right) \\
& +\sum_{k_{\alpha}>i, x_{\alpha} \in I(t)}\left|\sigma_{\alpha}\right| \cdot\left(\frac{\dot{x}_{\alpha}-\dot{a}}{m}-\frac{\left(x_{\alpha}-a\right) \dot{m}}{m^{2}}\right) \\
\geq & \sum_{k_{\alpha} \neq i}\left|\sigma_{\alpha}\right| \cdot \frac{c_{o}}{m(t)},
\end{aligned}
$$

thanks to the system's strict hyperbolicity and the non-resonance condition (1.4). In particular, observe that $c_{o} \leq c$ in (3.6). The above estimate yields a bound valid for all but finitely many times $t$ :

$$
K(t) \leq \frac{1}{c_{o}} \dot{\Phi}(t) m(t) .
$$

We notice again, as in [1 2], that there is a need for a completely different theory in order to tackle resonant cases, see also [13.

Concerning the term $M(t)$, observe that it can change only when an interaction occurs within the interval $[a(t), b(t)]$. In this case, one has

$$
M(\tau+)-M(\tau-)=\mathcal{O}(1)[Q(u(\tau-))-Q(u(\tau+))] .
$$

This yields an estimate of the form

$$
M(T)-M(t)=\mathcal{O}(1) \sum_{\tau \in \mathcal{T}}[Q(u(\tau-))-Q(u(\tau+))]
$$


where the summation extends over all times $\tau \in] 0, T]$ and where an interaction occurs inside $[a(\tau), b(\tau)]$. Inserting the estimates (3.7) - (3.8) in (3.4), we obtain

$$
\dot{m}(t)+C \dot{\Phi}(t) m(t) \geq M(T)-C\left(\epsilon+\sum_{\tau \in \mathcal{T}}|\Delta Q(\tau)|\right),
$$

for some constant $C$ and a.e. $t$. We now observe that $m$ is a continuous, piecewise linear function of $t$, and $\Phi$ is uniformly bounded. It can decrease only at interaction times, where 3.5 holds. Hence its total variation is uniformly bounded, and for some constant $K_{o}$ we have the estimate

$$
\int_{0}^{T} \dot{\Phi}(t) d t \leq K_{o}
$$

As in [3], from (3.9) we deduce the decay estimate:

$$
M(T) \leq 2 e^{C K_{o}}\left(\frac{b-a}{T}\right)+2 C \epsilon+2 C \sum_{\tau \in \mathcal{T}}|\Delta Q(\tau)| .
$$

II. Repeating the above process for any finite number $S$ of disjoint half-open intervals $I_{s} \doteq\left[a_{s}, b_{s}[\right.$, we obtain

$$
\sum_{s=1}^{S} M_{s}(T) \leq C^{\prime}\left(\sum_{s=1}^{S} \frac{b_{s}-a_{s}}{T}+S \epsilon+[Q(u(0))-Q(u(T))]\right),
$$

for some constant $C^{\prime}$ independent of $S$ and of the particular $(\epsilon, h)$-approximate solution. Here we have used the notation

$$
M_{s}(T) \doteq \sum_{k_{\alpha}=i, x_{\alpha} \in\left[a_{s}, b_{s}[\right.} \sigma_{\alpha}=\mu_{T}^{i}\left(\left[a_{s}, b_{s}[)\right.\right.
$$

to denote the sum of (signed) strength of all $i$-waves in $u(T, \cdot)$ contained in the interval $\left[a_{s}, b_{s}[\right.$.

III. Let us consider now any open interval $] a, b[$. Let $N$ be the number of $i$ shocks of the first generation in the front-tracking $(\epsilon, h)$-approximate solution $u$, as defined in [3], Chapter 7. We can thus construct half-open intervals $I_{s} \doteq\left[a_{s}, b_{s}[\right.$, $s=1, \ldots, S \leq N+1$, such that the following holds (see [3, Chapter 10):

- Every $i$-rarefaction front in $u(T, \cdot)$ contained in $] a, b[$ falls inside one of the intervals $I_{s}$.

- No $i$-shock front of the first generation falls inside any of the intervals $I_{s}$. Calling $\mu_{T}^{i+}$ the measure of positive $i$-waves in $u(T, \cdot)$, the above properties imply that

$$
\mu_{T}^{i+}(] a, b[)=\sum_{s} M_{s}(T)+\mathcal{O}(1) \cdot\left[\mathbf{Q}_{h}(u(0))-\mathbf{Q}_{h}(u(T))\right]+\mathcal{O}(1) \cdot r(\epsilon),
$$

where $\mathbf{Q}_{h}$ is the interaction potential introduced in [1], Section 4.1.

Indeed, the only negative $i$-waves contained in $\bigcup_{s} I_{s}$ must have generation order $\geq 2$, originating from interactions during the time interval $] 0, T]$. The total strength of these negative $i$-waves is bounded by the decrease in the interaction potential $Q$. The last term on the right-hand side of (3.13) tends to zero as $\epsilon$ does, and comes from the difference between $Q(u)$ and $\mathbf{Q}_{h}(u)$ : in the latter there are no non-physical fronts and all the countable $h$-Riemann problems are solved (whereas before only zero-waves inside the interval $(-1 / \epsilon, 1 / \epsilon)$ were considered). 
Altogether, (3.12) and (3.13) yield

$$
\mu_{T}^{i+}(] a, b[) \leq C^{\prime \prime}\left(\frac{b-a}{T}+(N+1) r(\epsilon)+\left[\mathbf{Q}_{h}(u(0))-\mathbf{Q}_{h}(u(T))\right]\right)
$$

for some constant $C^{\prime \prime}$ independent of $\epsilon$.

IV. For $h>0$ fixed, we now consider a sequence of $\left(\epsilon_{\nu}, h\right)$-approximate solutions satisfying the properties (i)-(iv) stated at the beginning of this section. It is clearly not restrictive to take $C^{\prime \prime} \geq C_{o}$ in (3.14), where $C_{o}$ is the (big) constant in Theorem 2.1. Using (2.8), (2.10), (3.14), (3.1) and (3.2), we obtain

$$
\begin{aligned}
& \mu_{T}^{i+}(] a, b[) \\
& \leq \liminf _{\nu \rightarrow \infty}\left(\mu_{\nu, T}^{i+}(] a, b[)+C^{\prime \prime} \mathbf{Q}_{h}\left(u_{\nu}(T)\right)\right)-C^{\prime \prime} \mathbf{Q}_{h}(u(T)) \\
& \leq C^{\prime \prime} \cdot \liminf _{\nu \rightarrow \infty}\left(\frac{b-a}{T}+\left(N_{\nu}+1\right) r(\epsilon)+\mathbf{Q}_{h}\left(u_{\nu}(0)\right)\right)-C^{\prime \prime} \mathbf{Q}_{h}(u(T)) \\
& \leq C^{\prime \prime} \cdot \frac{b-a}{T}+C^{\prime \prime}\left[\mathbf{Q}_{h}(u(0))-\mathbf{Q}_{h}(u(T))\right] \\
& \leq C^{\prime \prime} \cdot \frac{b-a}{T}+C^{\prime \prime}\left[\mathbf{Q}(u(0))-\mathbf{Q}(u(T))+\|\omega\|_{\mathbf{L}^{1}} \cdot \mathbf{V}(u(0))\right]
\end{aligned}
$$

Since the last term is independent of $h$, this proves (1.7) in case $J$ is an open interval. The same arguments can be used in the case where $J$ is a finite collection of open intervals. Since $\mu^{i}$ is a bounded Radon measure, the estimate (1.7) holds for every Borel set $J$, and we are done.

\section{A short COMment}

All these computations heavily rely on the restrictive assumption that the source term is dominated by a function $\omega \in \mathbf{L}^{1}(\mathbb{R})$ (the $\mathbf{L}^{\infty}(\mathbb{R})$ bound being just a consequence of the smoothness of $g$ ). In the case of a convex scalar law, [1],

$$
u_{t}+f(u)_{x}=g(u), \quad u_{o} \in \mathbf{L}^{1} \cap \mathbf{B V}(\mathbb{R}),
$$

the stability of the approximation procedure for (1.3) can be obtained requiring only $\omega \in \mathbf{L}^{\infty}(\mathbb{R})$. Hence one can follow the same canvas, and (3.4) simplifies a lot

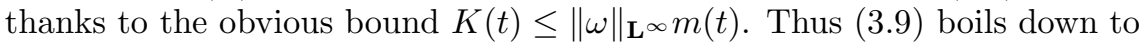

$$
\dot{m}(t)+\|\omega\|_{\mathbf{L} \infty m(t) \geq M(T),}
$$

which leads, in sharp contrast, to an exponential bound of the type $\left(\mu^{1}=u_{x}\right)$ :

$$
\mu_{t}^{1+}(J) \leq C e^{\|\omega\|_{\mathbf{L} \infty t}} \cdot \frac{\operatorname{meas}(J)}{t}, \quad t>0 .
$$

This agrees of course with simple computations, since in this context Oleinnik's estimates can be derived from the Riccati differential equation:

$$
z_{t}-g^{\prime}(u) z+f^{\prime \prime}(u) z^{2}=0, \quad z(0)=\sup _{\mathbb{R}}\left\{\max \left(0,\left(u_{o}\right)_{x}\right)\right\} \in \overline{\mathbb{R}} .
$$

Therefore one recovers (4.1) in the case $C=1 / \kappa$ and $\omega \equiv \operatorname{Lip}(g)$, since we have

$$
z(t)=\frac{e^{L i p(g) \cdot t}}{\frac{1}{z(0)}+\kappa\left(\frac{e^{L i p(g) \cdot t}-1}{L i p(g)}\right)} \leq \frac{e^{L i p(g) \cdot t}}{\kappa t}, \quad t>0 .
$$


All in all, the $\mathbf{L}^{1}(\mathbb{R})$ bound on $\omega$ expresses somehow the fact that the source has negligible effects outside a compact interval in $\mathbb{R}$, as pointed out in [8], p. 329 . Hence, by the strict hyperbolicity ensured by (1.4)-(3.6), waves exit this region after some time and then are ruled only by the convective process.

\section{ACKNOWLEDGMENTS}

Both authors acknowledge the hospitality of the Mathematics Department at the University of Pavia where this research was conducted. We also thank Prof. A. Bressan for constructive comments.

\section{REFERENCES}

1. D. Amadori, L. Gosse, and G. Guerra, Global BV entropy solutions and uniqueness for hyperbolic systems of balance laws, Arch. Rational Mech. Anal. 162 (2002), 327-366. MR 2003b:35127

2. D. Amadori, L. Gosse, and G. Guerra, Godunov-type approximation for a general resonant conservation law with large data, to appear in J. Differential Equations (2002).

3. A. Bressan, Hyperbolic systems of conservation laws - The one-dimensional Cauchy problem, Oxford University Press, 2000. MR 2002d:35002

4. A. Bressan and G. M. Coclite, On the boundary control of systems of conservation laws, SIAM J. Control Optim. 41 (2002), no. 2, 607-622. MR 2003f:93007

5. A. Bressan and R. M. Colombo, Decay of positive waves in nonlinear systems of conservation laws, Ann. Scuola Norm. Sup. Pisa 26 (1998), 133-160. MR 99e:35136

6. A. Bressan and P. Goatin, Olěnnik type estimates and uniqueness for $n \times n$ conservation laws, J. Differential Equations 156 (1999), 26-49. MR 2000d:35134

7. A. Bressan and P. Goatin, Stability of $\mathbf{L}^{\infty}$ solutions of Temple class systems, Differential Integral Equations 13 (2000), 1503-1528. MR 2001m:35209

8. C. M. Dafermos, Hyperbolic conservation laws in continuum physics, Grundlehren der Mathematischen Wissenschaften, Band 325, Springer-Verlag, Berlin, 2000. MR 2001m:35212

9. J. Glimm, Solutions in the large for nonlinear hyperbolic systems of equations, Comm. Pure Appl. Math. 18 (1965), 697-715. MR 33:2976

10. P. Goatin, One-sided Estimates and Uniqueness for Hyperbolic Systems of Balance Laws, Math. Models Methods in Applied Sci. 13 (2003) 527-543.

11. L. Gosse, A priori error estimate for a well-balanced scheme designed for inhomogeneous scalar conservation laws, C. R. Acad. Sci. Paris Sér. I Math. 327 (1998), no. 5, 467-472. MR 99h:35126

12. L. Gosse, Using K-branch entropy solutions for multiphase geometric optics computations, J. Comput. Phys. 180 (2002), no. 1, 155-182. MR 2003d:35250

13. S. Y. Ha, $\mathbf{L}^{1}$ stability for systems of conservation laws with a nonresonant moving source, SIAM J. Math. Anal. 33 (2001), no. 2, 411-439. MR 2002h:35178

14. D. Hoff, The sharp form of Oleinik's entropy condition in several space variables, Trans. Amer. Math. Soc. 276 (1983), no. 2, 707-714. MR 84b:35080

15. P. D. Lax, Hyperbolic systems of conservation laws, II, Comm. Pure Appl. Math. 10 (1957), 537-566. MR 20:176

16. P. G. LeFloch and A. E. Tzavaras, Representation of weak limits and definition of nonconservative products, SIAM J. Math. Anal. 30 (1999), no. 6, 1309-1342. MR 2001e:35113

17. T. P. Liu, Admissible solutions of hyperbolic conservation laws, Amer. Math. Soc. Memoir 38, no. 240 (1981). MR 82i:35116

18. O. Oleı̆nik, Discontinuous solutions of non-linear differential equations, Uspehi Mat. Nauk $\mathbf{1 2}$ (1957), 3-73. [English transl., Amer. Math. Soc. Transl. (2) 26 (1963), 95-172] MR 20:1055. MR 27:1721 
19. N. H. Risebro, A front-tracking alternative to the random choice method, Proc. Amer. Math. Soc. 117 (1993), no. 4, 1125-1139. MR 93e:35071

20. E. Tadmor, Local error estimates for discontinuous solutions of nonlinear hyperbolic equations, SIAM J. Numer. Anal. 28 (1991), no. 4, 891-906. MR 92d:35190

Centre de Mathématiques Appliquées, Ecole Polytechnique, 91128 Palaiseau Cedex, FRANCE

E-mail address: goatin@cmap.polytechnique.fr

Istituto per le Applicazioni del Calcolo (sezione di Bari), via G. Amendola, 122/I, 70126 BARI, ITALY

E-mail address: 1.gosse@area.ba.cnr.it 